DisCRETE ANALYSis, 2016:7, 19 pp.

www.discreteanalysisjournal.com

\title{
A Sharp Threshold for van der Waerden's Theorem in Random Subsets*
}

\author{
Ehud Friedgut ${ }^{\dagger}$ Hiệp Hàn ${ }^{\ddagger}$ Yury Person Mathias Schacht§
}

Received 21 December 2015; Published 29 February 2016

\begin{abstract}
We establish sharpness for the threshold of van der Waerden's theorem in random subsets of $\mathbb{Z} / n \mathbb{Z}$. More precisely, for $k \geqslant 3$ and $Z \subseteq \mathbb{Z} / n \mathbb{Z}$ we say $Z$ has the van der Waerden property if any two-colouring of $Z$ yields a monochromatic arithmetic progression of length $k$. Rödl and Ruciński (1995) determined the threshold for this property for any $k$ and we show that this threshold is sharp.

The proof is based on Friedgut's criterion (1999) for sharp thresholds and on the recently developed container method for independent sets in hypergraphs by Balogh, Morris and Samotij (2015) and by Saxton and Thomason (2015).
\end{abstract}

Key words and phrases: sharp thresholds, van der Waerden theorem

\section{Introduction}

One of the main research directions in extremal and probabilistic combinatorics over the last two decades has been the extension of classical results for discrete structures to the sparse random setting. Prime examples include Ramsey's theorem for graphs and hypergraphs $[2,8,9]$, Turán's theorem in extremal graph theory, and Szemerédi's theorem on arithmetic progressions $[2,12]$ (see also $[1,3,11]$ ). Results of that form establish the threshold for the classical result in the random setting. For a property the threshold is given by a function $\hat{p}=\hat{p}(n)$ such that for

\footnotetext{
${ }^{*}$ The cooperation of the authors was supported by the German-Israeli Foundation for Scientific Research and Development.

${ }^{\dagger}$ Supported by ISF grant 0398246.

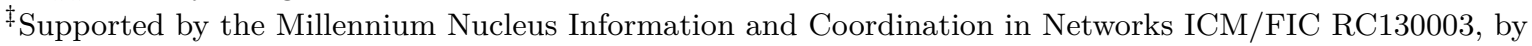
FONDECYT Iniciación grant 11150913, and by FAPESP (Proc. 2103/03447-6).

$\S$ Supported through the Heisenberg-Programme of the DFG.
} 


\section{Ehud Friedgut, Hiep Han, Yury Person, And Mathias Schacht}

every $p_{0} \ll \hat{p}$ the random graph $G\left(n, p_{0}\right)$ (or a random binomial subset of $[n]=\{1,2, \ldots, n\}$ ) with parameter $p_{0}$, the probability the property holds is asymptotically zero, whereas if $p_{0}$ is replaced by some $p_{1} \gg \hat{p}$ the property does hold asymptotically almost surely (a.a.s.), i.e., for a property $\mathcal{P}$ of graphs and probabilities $p=p(n)$ we have

$$
\lim _{n \rightarrow \infty} \mathbb{P}(G(n, p) \in \mathcal{P})= \begin{cases}0, & \text { if } p \ll \hat{p} \\ 1, & \text { if } p \gg \hat{p}\end{cases}
$$

The two statements involving $p_{0}$ and $p_{1}$ are referred to as the 0 -statement and the 1 -statement. For the properties mentioned above it can be shown that the optimal parameters $p_{0}$ and $p_{1}$ for which the 0 -statement and the 1-statement hold, only differ by a multiplicative constant.

The threshold for van der Waerden's theorem [15] is such an example and was obtained by Rödl and Ruciński in $[9,10]$. We denote by $[n]_{p}$ the binomial random subset of $[n]$, where every element of $[n]$ is included independently with probability $p=p(n)$. Furthermore, for a subset $A \subseteq[n]$ we write $A \rightarrow(k-\mathrm{AP})_{r}$ to denote the fact that no matter how one colours the elements of $A$ with $r$ colours there is always a monochromatic arithmetic progression with $k$ elements in $A$.

Theorem 1 (Rödl \& Ruciński). For every $k \geqslant 3$ and $r \geqslant 2$ there exist constants $c_{0}, c_{1}>0$ such that

$$
\lim _{n \rightarrow \infty} \mathbb{P}\left([n]_{p} \rightarrow(k-A P)_{r}\right)= \begin{cases}0, & \text { if } p \leqslant c_{0} n^{-\frac{1}{k-1}} \\ 1, & \text { if } p \geqslant c_{1} n^{-\frac{1}{k-1}}\end{cases}
$$

For the corresponding result in $\mathbb{Z} / n \mathbb{Z}$ and for two colours we close the gap between $c_{0}$ and $c_{1}$. More precisely, we show that there exist bounded sequences $c_{0}(n)$ and $c_{1}(n)$ with ratio tending to 1 as $n$ tends to infinity such that the statement holds (see Theorem 2 below). In other words, we establish a sharp threshold for van der Waerden's theorem for two colours in $\mathbb{Z} / n \mathbb{Z}$.

Similarly to the situation for subsets of $[n]$ we write $A \rightarrow(k-\mathrm{AP})_{r}$ for subsets $A \subseteq \mathbb{Z} / n \mathbb{Z}$ if any $r$-colouring of $A$ yields a monochromatic arithmetic progression with $k$-elements in $\mathbb{Z} / n \mathbb{Z}$ and we write $A \nrightarrow(k \text {-AP })_{r}$ if $A$ fails to have this property. Moreover, we denote by $\mathbb{Z}_{n, p}$ the binomial random subset of $\mathbb{Z} / n \mathbb{Z}$ with parameter $p$. With this notation at hand we can state our main result.

Theorem 2. For all $k \geqslant 3$ there exist constants $c_{1}>c_{0}>0$ and a function $c(n)$ satisfying $c_{0} \leqslant c(n) \leqslant c_{1}$ such that for every $\varepsilon>0$ we have

$$
\lim _{n \rightarrow \infty} \mathbb{P}\left(\mathbb{Z}_{n, p} \rightarrow(k-A P)_{2}\right)= \begin{cases}0, & \text { if } p \leqslant(1-\varepsilon) c(n) n^{-\frac{1}{k-1}} \\ 1, & \text { if } p \geqslant(1+\varepsilon) c(n) n^{-\frac{1}{k-1}}\end{cases}
$$

We have to insist on the setting of $\mathbb{Z} / n \mathbb{Z}$ (instead of $[n]$ ) since the symmetry will play a small but crucial rôle in our proof. Another shortcoming is the restriction to two colours $r=2$ and we believe it would be very interesting to extend the result to arbitrary $r$. We remark that only a few sharp thresholds for Ramsey properties are known (see, e.g., [6,7]) so far. 


\section{SHARP THRESHOLD FOR VAN DER WAERDEN'S THEOREM IN RANDOM SUBSETS}

Among other tools our proof relies heavily on the criterion for sharp thresholds of Friedgut and its extension due to Bourgain [4]. Another crucial tool is the recent container theorem for independent sets in hypergraphs due to Balogh, Morris and Samotij [1] and Thomason and Saxton [11].

Our proof extends to other Ramsey properties for two colours, as long as the corresponding extremal problem is degenerate, i.e., positive density yields many copies of the target structure and the target structure is strictly balanced with respect to its so-called 2-density. For example, even cycles in graphs, complete $k$-partite, $k$-uniform hypergraphs, and strictly balanced, density regular Rado systems (see [10]) satisfy these assumptions. Moreover, Schacht and Schulenburg [13] noted that the approach undertaken here can be refined to give a shorter proof for the sharp threshold of the Ramsey property for triangles and two colours from [7] and, more generally, for arbitrary odd cycles.

\section{Locality of coarse thresholds}

In [4] Friedgut gave a necessary condition for a graph property to have a coarse threshold, namely, that it is approximable by a "local" property. In the appendix to this work Bourgain proved a similar result for more general discrete structures. Here we state the special case applicable for properties in $\mathbb{Z} / n \mathbb{Z}$.

Theorem 3 (Bourgain). There exist functions $\delta(C, \tau)$ and $K(C, \tau)$ such that the following holds. Let $p=o(1)$ as $n$ tends to infinity, let $\mathcal{A}$ be a monotone family of subsets of $\mathbb{Z} / n \mathbb{Z}$, with

$$
\tau<\mu(p, \mathcal{A}):=\mathbb{P}\left(\mathbb{Z}_{n, p} \in \mathcal{A}\right)<1-\tau,
$$

and assume also $p \cdot \frac{d \mu(p, \mathcal{A})}{d p} \leqslant C$. Then there exists some $B \subseteq \mathbb{Z} / n \mathbb{Z}$ with $|B| \leqslant K$ such that

$$
\mathbb{P}\left(\mathbb{Z}_{n, p} \in \mathcal{A} \mid B \subseteq \mathbb{Z}_{n, p}\right)>\mathbb{P}\left(\mathbb{Z}_{n, p} \in \mathcal{A}\right)+\delta
$$

Note that whenever a property $\mathcal{A}$ (or rather, a series of properties $\mathcal{A}_{n}$ ) has a coarse threshold there exist constants $C$ and $\tau$ such that for infinitely many values of $n$ the hypothesis of the theorem holds. For applications, it would be problematic if there exists a $B$ with $|B| \leqslant K$ and $B \in \mathcal{A}$, since this would trivialise the conclusion (1). However, as observed in [5], the above theorem can be strengthened, without modifying the original proof, to deduce that the set of $B$ 's for which the assertion holds has non-negligible measure, i.e., there exists a family $\mathcal{B}$ such that

$$
\mathbb{P}\left(B \subseteq \mathbb{Z}_{n, p} \text { for some } B \in \mathcal{B}\right)>\eta,
$$

where $\eta>0$ depends only on $C$ and $\tau$ but not on $n$, and every $B \in \mathcal{B}$ satisfies the conclusion of Theorem 3, i.e., $|B| \leqslant K$ and

$$
\mathbb{P}\left(\mathbb{Z}_{n, p} \in \mathcal{A} \mid B \subseteq \mathbb{Z}_{n, p}\right)>\mathbb{P}\left(\mathbb{Z}_{n, p} \in \mathcal{A}\right)+\delta .
$$

This allows us to make assumptions about $B$ in the application below, as long as the set of $B$ 's violating the assumptions has negligible measure. In particular, Lemma 4 below implies 


\section{Ehud Friedgut, Hiep Han, Yury Person, And Mathias Schacht}

that any collection of sets $B \subseteq \mathbb{Z} / n \mathbb{Z}$, each of bounded size and with $B \rightarrow(k \text {-AP })_{2}$, appear only with probability tending to zero in $\mathbb{Z}_{n, p}$ for $p=O\left(n^{-\frac{1}{k-1}}\right)$. Consequently, in our proof we can therefore assume that the set $B$, provided by Theorem 3 on the assumption that $\mathbb{Z}_{n, p} \rightarrow(k \text {-AP })_{2}$ has a coarse thresholds, itself fails to have the van der Waerden property, i.e., $B \rightarrow(k-\mathrm{AP})_{2}$.

Lemma 4. Let $\mathcal{B}$ be a family of subsets of $\mathbb{Z} / n \mathbb{Z}$ with the property that every $B \in \mathcal{B}$ satisfies $|B| \leqslant \log \log n$ and $B \rightarrow(k-A P)_{2}$. Then for every $c>0$ and every sequence of probabilities $p=p(n) \leqslant c n^{-\frac{1}{k-1}}$ we have $\mathbb{P}\left(B \subseteq \mathbb{Z}_{n, p}\right.$ for some $\left.B \in \mathcal{B}\right)=o(1)$.

Lemma 4 was implicitly proved in [10, Section 7] (see the Deterministic and the Probabilistic Lemma there). For completeness we include a sketch of the proof here.

Proof (Sketch). Let $k \geqslant 3$ be an integer and let $p=p(n) \leqslant c n^{-1 /(k-1)}$ for some $c \in \mathbb{R}_{>0}$. For a set $Z \subseteq \mathbb{Z} / n \mathbb{Z}$ we consider the auxiliary $k$-uniform hypergraph $H_{Z, k}$ with vertex set $Z$ and hyperedges corresponding to $k$-APs in $Z$. The Deterministic Lemma [10, p. 500] asserts that if $Z \rightarrow(k-\mathrm{AP})_{2}$, then one of the following configurations must appear as a subhypergraph of $H_{Z, k}$ :

(i) either $H_{Z, k}$ contains a subhypergraph of type $\mathcal{T}_{1}$ consisting of a hyperedge $e_{0}$ and a loose cycle $C_{\ell}$ of some length $\ell \geqslant 3$, i.e., $C_{\ell}$ consists of $\ell$ hyperedges $e_{1}, \ldots, e_{\ell}$ satisfying for every $1 \leqslant i<j \leqslant \ell$

$$
\left|e_{i} \cap e_{j}\right|= \begin{cases}1, & \text { if } j=i+1 \text { or }(i=1 \text { and } j=\ell) \\ 0, & \text { otherwise }\end{cases}
$$

while the additional hyperedge $e_{0}$ has at least one vertex outside the cycle and shares at least two vertices with the cycles, i.e.,

$$
2 \leqslant\left|e_{0} \cap V\left(C_{\ell}\right)\right|<k
$$

(ii) or $H_{Z, k}$ contains a subhypergraph of type $\mathcal{T}_{2}$ consisting of a non-induced loose path $P_{\ell}$ of some length $\ell \geqslant 2$, i.e., $\ell$ hyperedges $e_{1}, \ldots, e_{\ell}$ satisfying for $1 \leqslant i<j \leqslant \ell$

$$
\left|e_{i} \cap e_{j}\right|= \begin{cases}1, & \text { if } j=i+1 \\ 0, & \text { otherwise } .\end{cases}
$$

The condition that $P_{\ell}$ is non-induced means that there exists some hyperedge $e_{0}$ in $E\left(H_{Z, k}\right) \backslash E\left(P_{\ell}\right)$ such that $e_{0} \subseteq V\left(P_{\ell}\right)$.

A simple first moment argument shows that a.a.s. no hypergraph on at most $\log n$ vertices of types $\mathcal{T}_{1}$ or $\mathcal{T}_{2}$ appears in $H_{\mathbb{Z}_{n, p}, k}$. For that let $X_{1}$ (resp. $X_{2}$ ) be the random variable counting the number of copies of hypergraphs of type $\mathcal{T}_{1}\left(\right.$ resp. $\left.\mathcal{T}_{2}\right)$ on at most $\log n$ vertices in $\mathbb{Z}_{n, p}$. Below we show that there exists a constant $K=K(k, c)$ such that

$$
\mathbb{E} X_{1} \leqslant(\log n)^{K} \cdot p \quad \text { and } \quad \mathbb{E} X_{2} \leqslant(\log n)^{K} \cdot p
$$

and since $p \leqslant c n^{-1 /(k-1)} \ll(\log n)^{-K}$ the lemma follows from Markov's inequality. 


\section{SHARP THRESHOLD FOR VAN DER WAERDEN'S THEOREM IN RANDOM SUBSETS}

We start with the random variable $X_{1}$. Since the hyperedges of $H_{\mathbb{Z}_{n, p}, k}$ correspond to $k$-APs the number $Y_{\ell}$ of loose cycles $C_{\ell}$ of length at least $\ell \geqslant 3$ in $H_{\mathbb{Z}_{n, p}, k}$ satisfies

$$
\mathbb{E} Y_{\ell} \leqslant O\left(p^{(k-1) \ell} \cdot n^{\ell}\right)=O\left(c^{(k-1) \ell} n^{-\ell} \cdot n^{\ell}\right)=O\left(c^{k \ell}\right) .
$$

For a given loose cycle $C_{\ell}$ the additional hyperedge $e_{0}$ (to complete $C_{\ell}$ to a hypergraph of type $\left.\mathcal{T}_{1}\right)$ shares at least two vertices with $C_{\ell}$. However, with these two vertices fixed there are less than $k^{2}$ possibilities to complete this choice to a $k$-AP in $\mathbb{Z} / n \mathbb{Z}$, i.e., these two vertices can be completed in at most $k^{2}$ ways to form the hyperedge $e_{0}$ in $H_{\mathbb{Z} / n \mathbb{Z}, k}$. In other words, for a fixed loose cycle $C_{\ell}$ on $(k-1) \ell$ vertices there are at most $(k-1)^{2} \ell^{2} \cdot k^{2}$ possibilities to complete $C_{\ell}$ to a hypergraph of type $\mathcal{T}_{1}$. Furthermore, since the hyperedge $e_{0}$ is required to have at least one vertex outside $C_{\ell}$ we have

$$
\mathbb{E} X_{1}<\sum_{\ell=3}^{\log \log n} k^{4} \ell^{2} p \cdot \mathbb{E} Y_{\ell}=\sum_{\ell=3}^{\log \log n} O\left(\ell^{2} p \cdot c^{k \ell}\right) \leqslant(\log n)^{K} \cdot p
$$

for some constant $K=K(k, c)$, which establishes the first estimate in (2).

Similarly, for the random variable $X_{2}$ we first observe that the expected number $Y_{\ell}^{\prime}$ of loose paths $P_{\ell}$ of length at least $\ell \geqslant 2$ in $\mathbb{Z}_{n, p}$ satisfies

$$
\mathbb{E} Y_{\ell}^{\prime} \leqslant O\left(p^{k} n^{2} \cdot p^{(k-1)(\ell-1)} n^{(\ell-1)}\right)=O\left(p n \cdot c^{k \ell}\right) .
$$

Since the additional hyperedge $e_{0}$ reduces the expected number of choices for at least one of the hyperedges of $P_{\ell}$ from $O\left(p^{k-1} n\right)$ to $O\left(p^{k-1}\right)$ and since $e_{0}$ is fixed after selecting two of its vertices within $P_{\ell}$ we arrive at

$$
\mathbb{E} X_{2} \leqslant \sum_{\ell=2}^{\log \log n} O\left(\frac{\ell^{2}}{n}\right) \cdot \mathbb{E} Y_{\ell}^{\prime}=\sum_{\ell=2}^{\log \log n} O\left(\ell^{2} p \cdot c^{k \ell}\right) \leqslant(\log n)^{K} \cdot p
$$

for some constant $K=K(k, c)$, which establishes the second estimate in (2) and concludes the proof of the lemma.

We summarise the discussion above in the following corollary of Theorem 3 , which is tailored for our proof of Theorem 2.

Corollary 5. Assume that the property $\left\{Z \subseteq \mathbb{Z} / n \mathbb{Z}: Z \rightarrow(k-A P)_{2}\right\}$ does not have a sharp threshold. Then there exist constants $c_{1}, c_{0}, \alpha, \varepsilon, \mu>0$, and $K$ and a function $c(n): \mathbb{N} \rightarrow \mathbb{R}$ with $c_{0}<c(n)<c_{1}$ so that for infinitely many values of $n$ and $p=c(n) n^{-\frac{1}{k-1}}$ the following holds.

There exists a subset $B$ of $\mathbb{Z} / n \mathbb{Z}$ of size at most $K$ with $B \nrightarrow(k-A P)_{2}$ such that for every family $\mathbb{Z}$ of subsets from $\mathbb{Z} / n \mathbb{Z}$ satisfying $\mathbb{P}\left(\mathbb{Z}_{n, p} \in \mathbb{Z}\right)>1-\mu$ there exists a $Z \in \mathbb{Z}$ so that

(a) $\mathbb{P}\left(Z \cup(B+x) \rightarrow(k-A P)_{2}\right)>\alpha$, where $x \in \mathbb{Z} / n \mathbb{Z}$ is chosen uniformly at random, and

(b) $\mathbb{P}\left(Z \cup \mathbb{Z}_{n, \varepsilon p} \rightarrow(k-A P)_{2}\right)<\alpha / 2$. 


\section{Ehud Friedgut, Hiep Han, Yury Person, And Mathias Schacht}

We remark that the $\mathbb{P}(\cdot)$ in Corollary 5 concern different probability spaces. While the assumption $\mathbb{P}\left(\mathbb{Z}_{n, p} \in \mathbb{Z}\right)>1-\mu$ concerns the binomial random subset $\mathbb{Z}_{n, p}$, we consider $x$ chosen uniformly at random from $\mathbb{Z} / n \mathbb{Z}$ in $(a)$ and the binomial random subset $\mathbb{Z}_{n, \varepsilon p}$ in $(b)$. We close this section with a short sketch of the proof of Corollary 5.

Proof (Sketch). For $k \geqslant 3$ we consider the property $\mathcal{A}=\left\{Z \subseteq \mathbb{Z} / n \mathbb{Z}: Z \rightarrow(k \text {-AP })_{2}\right\}$ and assume that it does not have a sharp threshold. Consequently, there exists a function $p=p(n)$ such that for infinitely many $n$ the assumptions of Theorem 3 hold, which implicitly yields constants $C$, $\tau, \delta$, and $K$. Let $\overline{\mathcal{A}}=\mathcal{P}(\mathbb{Z} / n \mathbb{Z}) \backslash \mathcal{A}$ be the family of subsets of $\mathbb{Z} / n \mathbb{Z}$ that fail to have the van der Waerden property. Since we assume that the threshold for $\mathcal{A}$ is not sharp, we may fix $\varepsilon>0$ sufficiently small, such that there must be some $\alpha$ with $\delta / 2>\alpha>0$ so that if we let $Z^{\prime} \subseteq \overline{\mathcal{A}}$ be the sets $Z \in \overline{\mathcal{A}}$ for which

$$
\mathbb{P}\left(Z \cup \mathbb{Z}_{n, \varepsilon p} \rightarrow(k-\mathrm{AP})_{2}\right)<\alpha / 2
$$

then $\mathbb{P}\left(\mathbb{Z}_{n, p} \in \mathbb{Z}^{\prime} \mid \mathbb{Z}_{n, p} \in \overline{\mathcal{A}}\right) \geqslant 1-\delta / 4$.

Also for $p=p(n)$ we have $\tau<\mathbb{P}\left(\mathbb{Z}_{n, p} \rightarrow(k-\mathrm{AP})_{2}\right)=\mathbb{P}\left(\mathbb{Z}_{n, p} \in \mathcal{A}\right)<1-\tau$, so by Theorem 1 there

exist some constants $c_{1} \geqslant c_{0}>0$ such that $p=p(n)=c(n) n^{-\frac{1}{k-1}}$ for some function $c(n): \mathbb{N} \rightarrow \mathbb{R}$ satisfying $c_{0} \leqslant c(n) \leqslant c_{1}$. Strictly speaking, we should use the version of Theorem 1 for $\mathbb{Z} / n \mathbb{Z}$ instead of $[n]$. However, it is easy to see that the 1-statement for random subsets of $[n]$ implies the 1-statement for random subsets of $\mathbb{Z} / n \mathbb{Z}$ (up to a different constant $c_{1}$ ) and the proof of the 0 -statement from [10] can be straightforwardly adjusted for subsets of $\mathbb{Z} / n \mathbb{Z}$.

Moreover, for any such $n$ Theorem 3 yields a family $\mathcal{B}$ of subsets of $\mathbb{Z} / n \mathbb{Z}$ each of size at most $K$ such that (1) holds and an element of $\mathcal{B}$ appears as a subset of $\mathbb{Z}_{n, p}$ with probability at least $\eta$. Consequently, Lemma 4 asserts that at least one such $B \in \mathcal{B}$ fails to have the van der Waerden property itself, i.e., $B \nrightarrow(k \text {-AP })_{2}$. By symmetry it follows from (1), that the same holds for every translate $B+x$ with $x \in \mathbb{Z} / n \mathbb{Z}$. In particular, consider the family $Z^{\prime \prime} \subseteq \overline{\mathcal{A}}$ of all sets $Z \in \overline{\mathcal{A}}$ such that for at least $(\delta / 2) n$ translates $B+x$ we have $Z \cup(B+x) \rightarrow(k \text {-AP })_{2}$, i.e.,

$$
\mathbb{P}\left(Z \cup(B+x) \rightarrow(k-\mathrm{AP})_{2}\right)>\delta / 2>\alpha
$$

for $x$ chosen uniformly at random from $\mathbb{Z} / n \mathbb{Z}$. Then $\mathbb{P}\left(\mathbb{Z}_{n, p} \in \mathbb{Z}^{\prime \prime} \mid \mathbb{Z}_{n, p} \in \overline{\mathcal{A}}\right) \geqslant \delta / 2$. So, taking $\mu<\delta \cdot \mathbb{P}\left(\mathbb{Z}_{n, p} \in \overline{\mathcal{A}}\right) / 8$ we have that if $\mathbb{P}\left(\mathbb{Z}_{n, p} \in \mathbb{Z}\right) \geqslant 1-\mu$ then $Z \cap Z^{\prime} \cap Z^{\prime \prime} \neq \varnothing$. Any $Z$ in this non-empty family has the desired properties.

\section{Lemmas and the proof of the main theorem}

In this section we state all the necessary notation and lemmas to give the proof of Theorem 2. We start with an outline of this proof.

\subsection{Outline of the proof}

The point of departure is Corollary 5 and we will derive a contradiction to its second property. To this end, we consider an appropriate set $Z$ as given by Corollary 5 and let $\Phi$ denote the set of all colourings of $Z$ without a monochromatic $k$-AP. The main obstacle is to find a partition 


\section{SHARP THRESHOLD FOR VAN DER WAERDEN'S THEOREM IN RANDOM SUBSETS}

of $\Phi$ into $i_{0}=2^{o(p n)}$ classes $\Phi_{1}, \ldots, \Phi_{i_{0}}$, such that any two colourings $\varphi, \varphi^{\prime}$ from any partition class $\Phi_{i}$ agree on a relatively dense subset $C_{i}$ of $Z$, i.e. $\varphi(z)=\varphi^{\prime}(z)$ for all $z \in C_{i}$. Let $B_{i}$ denote the larger monochromatic subset of $C_{i}$, say of colour blue. We consider the the set $F\left(B_{i}\right)$ of those elements in $\mathbb{Z} / n \mathbb{Z}$, which extend a blue $(k-1)$-AP in $B_{i}$ to an $k$-AP. Note that Corollary 5 allows us to impose further conditions on $Z$ as long as $\mathbb{Z}_{n, p}$ satisfies them almost surely. One of these properties will assert that $F\left(B_{i}\right)$ is of size linear in $n$ Consequently, by a quantitative version of Szemerédi's theorem we know that the number of $k$-APs in the focus of $B_{i}$ is $\Omega\left(n^{2}\right)$. Consider $U_{i}=(\mathbb{Z} / n \mathbb{Z})_{\varepsilon p} \cap F\left(B_{i}\right)$ and note that if any element of $U_{i}$ is coloured blue then this induces a blue $k$-AP with $Z$ under any colouring $\varphi \in \Phi_{i}$. Hence, to extend any $\varphi \in \Phi_{i}$ to a colouring of $Z \cup U_{i}$ without a monochromatic $k$-AP it is necessary that all elements in $U_{i}$ are coloured red. Consequently, the probability of a successful extension of any colouring in $\Phi_{i}$ is bounded from above by the probability that $U_{i}$ does not contain a $k$-AP. This, however, is at most $\exp \left(-\Omega\left(p^{k} n^{2}\right)\right)=\exp (-\Omega(p n))$ by Janson's inequality. We conclude by the union bound that after the second round, i.e. $\mathbb{Z}_{n, \varepsilon p}$, the probability that any $k$-AP-free colouring of $Z$ survives is $i_{0} \exp (-\Omega(p n))=o(1)$ which contradicts $(b)$.

To establish the above mentioned partition of $\Phi$ we will define an auxiliary hypergraph $H$ in such a way that every $\varphi \in \Phi$ can be associated with a hitting set of $H$. As the complements of hitting sets are independent and as $H$ will be "well-behaved" we can apply a structural result of Balogh, Morris and Samotij [1] on independent sets in uniform hypergraphs (see Theorem 15) to "capture" the hitting sets of $H$ and hence a partition of $\Phi$ with the properties mentioned above (see Lemma 8). We remark, that the proof of showing that $H$ is indeed well-behaved will use $(a)$ and additional properties of $Z$, which hold a.a.s. in $\mathbb{Z}_{n, p}$. Next we will introduce the necessary concepts along with the lemmas needed to give the proof of the main theorem. The proof of Theorem 2 will then be given in Section 3.3.

\subsection{Lemmas}

We call $(Z, B)$ an interacting pair if $Z \nrightarrow(k \text {-AP })_{2}$ and $B \nrightarrow(k \text {-AP })_{2}$ but $Z \cup B \rightarrow(k \text {-AP })_{2}$. Further, $(Z, B, X)$ is called an interacting triple if $(Z, B+x)$ is interacting for all $x \in X$. Note that Corollary 5 asserts that there is an interacting triple $(Z, B, X)$ with $|X|>\alpha n$. In the following we shall concentrate on elements which are decisive for interactions. Given a (not necessarily interacting) pair $(A, B)$ we say that an element $a \in A$ focuses on $b \in B+x$ if there are $k-2$ further elements in $A \cup B$ forming a $k$-AP with $a$ and $b$.

The set of vertices of particular interest, given a pair $(A, B)$, is

$$
M(A, B)=\{a \in A \text { : there is a } b \in B \text { such that } a \text { focuses on } b\}
$$

and for a triple $(A, B, X)$ we define the hypergraph $H=H(A, B, X)$ with the vertex set $A$ and the edge set consisting of all $M(A, B+x)$ with $x \in X$. We are interested in the hypergraph $H(Z, B, X)$ with an interacting triple $(Z, B, X)$. We will make use of the fact that Corollary 5 allows us to put further restrictions on $Z$ as long these events occur a.a.s. for $\mathbb{Z}_{n, p}$. The requirement we want to make is that the maximum degree and co-degree of $H(Z, B, X)$ are well behaved. 


\section{Ehud Friedgut, Hiep Han, Yury Person, And Mathias Schacht}

Lemma 6. For given $c_{1}, k, K$ and all $B \subset \mathbb{Z} / n \mathbb{Z}$ of size $|B| \leqslant K$ the following holds a.a.s. for $2 \log n \leqslant p \leqslant c_{1} n^{-1 /(k-1)}$ : There is a set $Y \subset \mathbb{Z} / n \mathbb{Z}$ of size at most $n^{1-1 /(k-1)} \log n$ such that the hypergraph $H=H\left(\mathbb{Z}_{n, p}, B,(\mathbb{Z} / n \mathbb{Z}) \backslash Y\right)$ satisfies

(1) $2 p n \geqslant v(H) \geqslant p n / 2$,

(2) $\Delta_{1}(H) \leqslant 10 k^{3} K p^{k-2} n$, and

(3) $\Delta_{2}(H) \leqslant 8 \log n$,

where $\Delta_{1}(H)$ and $\Delta_{2}(H)$ denote the maximum vertex degree of $H$ and maximum co-degree of pairs of vertices of $H$.

We postpone the proof of Lemma 6. It can be found in Section 4 .

A set of vertices of a hypergraph is called a hitting set if it intersects every edge of this hypergraph. The conditions in Lemma 6 will be used to control the hitting sets of $H(Z, B, X)$ which play an important rôle as explained in the following. A colouring of a set is called $k$-AP free if it does not exhibit a monochromatic $k$-AP. For an interacting triple $(Z, B, X)$ we fix a $k$-AP free colouring $\sigma: B \rightarrow\{$ red,blue $\}$ of $B$, which exists since $B \nrightarrow(k \text {-AP })_{2}$. We also consider the "same" colouring for all its translates $B+x$. More precisely, let $B=\left\{b_{1}, \ldots, b_{|B|}\right\}$ and for every $x \in X$ we consider the $k$-AP free colouring $\sigma_{x}:(B+x) \rightarrow\{$ red,blue $\}$ defined by $\sigma_{x}\left(b_{i}+x\right)=\sigma\left(b_{i}\right)$.

For any $k$-AP-free colouring $\varphi$ of $Z$ and any $x \in X$ the colouring of $Z \cup(B+x)$ induced by $\sigma_{x}$ and $\varphi$ must exhibit a monochromatic $k$-AP (intersecting both $Z$ and $B+x$ ) since $(Z, B+x)$ is interacting. Hence, for each $x \in X$ the edge $M(Z, B+x)$ contains an element $z$ focussing on an element $b \in B+x$ such that $\varphi(z)=\sigma_{x}(b)$. Such a vertex $z \in M(Z, B+x)$ we call activated by $\sigma_{x}$ and $\varphi$ and we define the set of activated vertices

$$
A_{\varphi}^{\sigma_{x}}(Z, B+x)=\left\{z \in Z: z \text { is activated by } \sigma_{x} \text { and } \varphi\right\}
$$

which is a non-empty subset of $M(Z, B+x)$.

Observation 7. Suppose that we are given an interacting triple $(Z, B, X)$, a $k$-AP free colouring $\sigma: B \rightarrow\{$ red,blue $\}$ of $B=\left\{b_{1}, \ldots, b_{|B|}\right\}$, and suppose for every $x \in X$ the translate $B+x$ is coloured with the same pattern $\sigma_{x}$. Further, let $\varphi$ be a $k$-AP free colouring of $Z$. Then the set of activated vertices

$$
A_{\varphi}=A_{\varphi}^{\sigma}(Z, B, X)=\bigcup_{x \in X} A_{\varphi}^{\sigma_{x}}(Z, B+x)
$$

is a hitting set of $H(Z, B, X)$.

The following lemma shows that the hitting sets of well-behaved uniform hypergraphs can be "captured" by a small number of sets of large size called cores. 


\section{SHARP THRESHOLD FOR VAN DER WAERDEN'S THEOREM IN RANDOM SUbSETS}

Lemma 8. For every natural $k \geqslant 3, \ell \geqslant 2$ and all positive $C_{0}, C_{1}$ there are $C^{\prime}$ and $\beta>0$ such that the following holds.

If $H$ is an $\ell$-uniform hypergraph with $m$ vertices, $C_{0} m^{1+1 /(k-2)}$ edges, $\Delta_{1}(H) \leqslant C_{1} m^{1 /(k-2)}$, and $\Delta_{2}(H) \leqslant C_{1} \log m$ then there is a family $\mathcal{C}$ of subsets of $V(H)$, which we shall call cores, such that

(i) for $t=1-1 /(k-2)(\ell-1)$ we have

$$
|\mathcal{C}| \leqslant \sum_{i=1}^{C^{\prime} m^{t}}\left(\begin{array}{c}
m \\
i
\end{array}\right)
$$

(ii) $|C| \geqslant \beta m$ for every $C \in \mathcal{C}$, and

(iii) every hitting set of $H$ contains some $C$ from $\mathcal{C}$.

Lemma 8 will follow from the main result from [1]. The proof can be found in Section 5 . As it turns out, we can insist that the interacting triple $(Z, B, X)$ guaranteed by Corollary 5 has the additional property that $X$ contains a suitable subset $X^{\prime} \subset X$ so that the hypergraph $H\left(Z, B, X^{\prime}\right)$ is uniform. In this case Lemma 8 allows us to partition the sets of all $k$-AP free colourings of $Z$ into a small number of partition classes $\left\{\Phi_{C}\right\}_{C \in \mathcal{C}}$, each represented by a big core $C \in \mathcal{C}$.

However, we wish to refine the partition classes further so that every two colourings $\varphi, \varphi^{\prime} \in \Phi_{C}$ from the same partition class agree on a large vertex set. This can be accomplished by applying Lemma 8 to $H\left(Z, B, X^{\prime}\right)$ for a more refined subset $X^{\prime} \subset X$. Indeed, we will make sure that there is a set which guarantees that the colours of the activated vertices $A_{\varphi}$ under $\varphi$ as defined in Observation 7 are already "determined" by $\sigma$. This implies that any two colourings $\varphi, \varphi^{\prime} \in \Phi_{C}$ agree on $A_{\varphi} \cap A_{\varphi^{\prime}}$, hence, on the core $C$ representing them, i.e. $\varphi(z)=\varphi^{\prime}(z)$ for all $z \in C$. To make this formal we need the following definitions.

In the following we fix some linear order on the elements of $\mathbb{Z} / n \mathbb{Z}$, which we denote simply by $<$. A triple $(Z, B, X)$ is called regular if for all $x \in X$ every element of $Z$ focuses on at most one element in $B+x$. Given a regular triple $(Z, B, X)$ and an $x \in X$ let $z_{1}<\cdots<z_{\ell}$ denote the elements of $M_{x}=M(Z, B+x) \in H(Z, B, X)$. We say that $z \in M(Z, B+x)$ has index $i$ if $z=z_{i}$ and the triple $(Z, B, X)$ is called index consistent if for any element $z \in Z$ and any two edges $M_{x}, M_{x^{\prime}}$ containing $z$ the indices of $z$ in $M_{x}$ and $M_{x^{\prime}}$ are the same.

Further, let $B=\left\{b_{1}, \ldots, b_{|B|}\right\}$. We associate to the edge $M_{x}$ its profile which is the function $\pi:[\ell] \rightarrow[|B|]$ indicating which $z_{i}$ focusses on which $b_{j}+x$, formally: $\pi(i)=j$ if $z_{i}$ focusses on $b_{j}+x$. Since $(Z, B, X)$ is regular, each $z \in M_{x}$ focuses on exactly one element from $B+x$, thus, the profile of $M_{x}$ is well-defined and unique. We call $\ell$ the length of the profile and we say that the triple $(Z, B, X)$ has profile $\pi$ with length $\ell$ if all edges of $H(Z, B, X)$ do. We summarise the desired properties for the hitting sets of $H(Z, B, X)$ associated to $k$-AP free colourings of $Z$. 
EhUd Friedgut, Hiep Han, Yury Person, And Mathias Schacht

Observation 9. Fix some linear order on $\mathbb{Z} / n \mathbb{Z}$. Suppose the triple $(Z, B, X)$ in Observation 7 with $B=\left\{b_{1}, \ldots, b_{|B|}\right\}$ is index consistent and has profile $\pi$. Let $A_{\varphi}=A_{\varphi}^{\sigma}(Z, B, X)$ be the vertex set activated by $\varphi$ and $\sigma$ as defined in Observation \%. Then for any vertex $z \in A_{\varphi}$ the colour $\varphi(z)$ of $z$ is already determined by $\sigma$ and the (unique) index $i$ of $z$, indeed, $\varphi(z)=\sigma\left(b_{\pi(i)}\right)$. In particular, any two $k$-AP free colourings $\varphi$ and $\varphi^{\prime}$ of $Z$ agree on $A_{\varphi} \cap A_{\varphi^{\prime}}$, i.e. $\varphi(z)=\varphi^{\prime}(z)$ for all $z \in A_{\varphi} \cap A_{\varphi^{\prime}}$.

The following lemma will allow us to restrict considerations to index consistent triples with a bounded length profile.

Lemma 10. For all $c_{1}>0, k, K$ and $\alpha>0$ there exist $L$ and $\alpha^{\prime}>0$ such that for all $B \subset \mathbb{Z} / n \mathbb{Z}$ of size $|B| \leqslant K$ and $p \leqslant c_{1} n^{-1 /(k-1)}$ the following holds a.a.s.

For any linear order on $\mathbb{Z} / n \mathbb{Z}$ there is a set $Y_{n} \subset \mathbb{Z} / n \mathbb{Z}$ of size at most $n^{1-1 /(k-1)} \log n$ such that for every set $X \subset \mathbb{Z} / n \mathbb{Z}$ of size $X \geqslant \alpha$ there is a set $X^{\prime} \subset X \backslash Y_{n}$ of size $\left|X^{\prime}\right| \geqslant \alpha^{\prime} n$ and a profile $\pi$ of length at most $L$ such that $\left(\mathbb{Z}_{n, p}, B, X^{\prime}\right)$ is index consistent and has profile $\pi$.

The proof of Lemma 10 can be found in Section 4. Lastly, we put another restriction on $Z$ as to make sure that any relatively dense subset of any core creates many $k$-AP's for the second round.

Lemma 11. For every $c_{0}>0$ and $\gamma>0$ there is a $\delta>0$ such that for $p \geqslant c_{0} n^{-1 /(k-1)}$ a.a.s. the following holds. The size of $\mathbb{Z}_{n, p}$ is at most 2 pn and for every subset $S \subset \mathbb{Z}_{n, p}$ of size $|S|>\gamma p n$ the set

$$
F(S)=\left\{z \in \mathbb{Z} / n \mathbb{Z}: \text { there are } a_{1}, \ldots, a_{k-1} \in S \text { which form a } k \text {-AP with } z\right\}
$$

has size at least $\delta n$.

The proof of Lemma 11 can be found in Section 6. We are now in the position to prove the main theorem.

\subsection{Proof of the main theorem}

The proof of the main theorem uses the lemmas introduced in the previous section and follows the scheme described.

Proof of Theorem 2. For a given $k \geqslant 3$ assume for contradiction that $\mathbb{Z}_{n, p} \rightarrow(k$-AP $)$ does not have a sharp threshold. By Corollary 5 there exist constants $c_{0}, c_{1}, \alpha, \varepsilon, \mu>0$ and $K$ and a function $p(n)=c(n) n^{-1 /(k-1)}$ for some functin $c(n)$ satisfying $c_{0} \leqslant c(n) \leqslant c_{1}$ such that for infinitely many $n$ there exists a subset $B \subset \mathbb{Z} / n \mathbb{Z}$ of size at most $K$ with $B \nrightarrow(k \text {-AP })_{2}$.

We apply Lemma 10 with $c_{1}, k, K$, and $\alpha$ to obtain $L$ and $\alpha^{\prime}>0$. For each $2 \leqslant \ell \leqslant L$ we apply Lemma 8 with the constants $k, \ell, C_{0}=\alpha^{\prime} /\left(2 c_{1}\right)$, and $C_{1}=2\left(10 k^{3} K\right)^{k-2} c_{1}^{(k-2)^{2}-1}$ to obtain $C^{\prime}(\ell)$ and $\beta(\ell)>0$. Let

$$
C^{\prime}=\max \left\{C^{\prime}(\ell): 2 \leqslant \ell \leqslant L\right\} \quad \text { and } \quad \beta=\min \{\beta(\ell): 2 \leqslant \ell \leqslant L\}
$$




\section{SHARP THRESHOLD FOR VAN DER WAERDEN'S THEOREM IN RANDOM SUBSETS}

and apply Lemma 11 with $c_{0}$ and $\gamma=\beta / 4$ to obtain $\delta>0$.

For each $n$ we define $Z_{n}$ to be the sets of subsets $Z \subset \mathbb{Z} / n \mathbb{Z}$ which satisfy the conclusions of Lemma 6, Lemma 10 and Lemma 11 (with $\mathbb{Z}_{n, p}$ replaced by $Z$ ) with the constants given and chosen from above. As these lemmas assert properties of $\mathbb{Z}_{n, p}$ that hold a.a.s. we know that for sufficiently large $n$ we have $\mathbb{P}\left(\mathbb{Z}_{n, p} \in Z_{n}\right)>1-\mu$. Hence, by Corollary 5 there is an interacting triple $(Z, B, X)$ such that $|B| \leqslant K,|X| \geqslant \alpha n$ and $Z \in Z_{n}$. In particular, since $Z$ satisfies the conclusion of Lemma 6 and Lemma 10 there exists a set $Y_{n}$ of size at most $2 n^{1-1 /(k-1)} \log n$ and a profile $\pi$ of length $1 \leqslant \ell \leqslant L$ and a set $X^{\prime} \subset X \backslash Y_{n}$ such that

- $\left|X^{\prime}\right| \geqslant \alpha^{\prime} n$

- the (interacting) triple $\left(Z, B, X^{\prime}\right)$ is index consistent and has profile $\pi$,

- the hypergraph $H=H\left(Z, B, X^{\prime}\right)$ satisfies $2 p n \geqslant v(H)=|Z| \geqslant p n / 2$, the maximum degree of $H$ satisfies $\Delta_{1}(H) \leqslant 10 k^{3} K p^{k-2} n$ and $\Delta_{2}(H) \leqslant 8 \log n$.

As $\left(Z, B, X^{\prime}\right)$ is regular we have $\ell \geqslant k-1 \geqslant 2$ by definition. Further, $H$ is an $\ell$-uniform hypergraph on $m=|Z|$ vertices which satisfies the assumptions of Lemma 8 with the constants chosen above. Hence, by the conclusions of Lemma 8 we obtain a family $\mathcal{C}$ of cores, such that

(i) for $t=1-1 /(k-2)(\ell-1)$ we have

$$
|\mathcal{C}|=\sum_{i=1}^{C^{\prime} m^{t}}\left(\begin{array}{c}
m \\
i
\end{array}\right)
$$

(ii) $|C| \geqslant \beta m$ for every $C \in \mathcal{C}$, and

(iii) every hitting set of $H$ contains some $C$ from $\mathcal{C}$.

Let $\Phi$ be the set of all $k$-AP free colourings of $Z$. By Observation 7 and Observation 9 we can associate to each $\varphi \in \Phi$ a hitting set $A_{\varphi}$ of $H$ such that any two colourings $\varphi, \varphi^{\prime} \in \Phi$ agree on $A_{\varphi} \cap A_{\varphi^{\prime}}$, i.e. $\varphi(z)=\varphi^{\prime}(z)$ for all $z \in A_{\varphi} \cap A_{\varphi^{\prime}}$. For any $C \in \mathcal{C}$ we define $\Phi_{C}$ to be the set of $\varphi \in \Phi$ such that $C \subset A_{\varphi}$ and obtain $\Phi=\bigcup_{C \in \mathcal{C}} \Phi_{C}$. Clearly, for any $C \in \mathcal{C}$, any two $\varphi, \varphi^{\prime} \in \Phi_{C}$ agree on $C \subset A_{\varphi} \cap A_{\varphi^{\prime}}$. Let $B_{C} \subset C$ be the larger monochromatic subset of $C$ under (any) $\varphi \in \Phi_{C}$, say of colour blue. Then $B_{C}$ has size $\left|B_{C}\right| \geqslant|C| / 2 \geqslant \gamma p n$ and as $Z \in Z_{n}$ we know by Lemma 11 that $\left|F\left(B_{C}\right) \backslash Z\right|>\delta n / 2$. Let $\mathcal{P}(C)$ denote the set of all $k$-APs contained in $F\left(B_{C}\right)$. By the quantitative version of Szemerédi's theorem (see [14]) we know that there is an $\eta>0$ such that for sufficiently large $n$ we have $|\mathcal{P}(C)| \geqslant \eta n^{2}$. Consider the second round exposure $U_{C}=\mathbb{Z}_{n, \varepsilon p} \cap F\left(B_{C}\right)$ and let $t_{i}$ be the indicator random variable for the event $i \in U_{C}$. We are interested in the probability that there is a $\varphi \in \Phi_{C}$ which can be extended to a $k$-AP free colouring of $Z \cup U_{C}$. To extend any colouring $\varphi \in \Phi_{C}$ of $Z$ to a $k$-AP free colouring of $Z \cup U_{C}$, however, it is necessary that $U_{C} \subset F\left(B_{C}\right)$ is completely coloured red, i.e. that $U_{C}$ does not contain any $k$-AP. This probability can be bounded using Janson's inequality for $X=\sum_{P \in \mathcal{P}(C)} \prod_{i \in P} t_{i}$ given by

$$
\mathbb{P}(X=0) \leqslant \exp \left\{-\frac{\mathbb{E}(X)^{2}}{2 \Delta}\right\}
$$




\section{Ehud Friedgut, Hiep Han, Yury Person, and Mathias Schacht}

where

$$
\Delta=\sum_{A, B \in \mathcal{P}(C): A \cap B \neq \varnothing} \mathbb{E}\left(\prod_{i \in A \cup B} t_{i}\right) \leqslant p^{2 k-1} n^{3}+p^{k+1} k^{2} n^{2} \leqslant 2 p^{2 k-1} n^{3}
$$

for large enough $n$. We obtain

$\mathbb{P}\left(\exists \varphi \in \Phi_{C}: \varphi\right.$ can be extended to a $k$-AP free colouring of $\left.Z \cup U_{C}\right)$

$$
\leqslant \mathbb{P}\left(U_{C} \text { does not contain a } k-\mathrm{AP}\right)<\exp \left\{-\eta^{2} p n / 4\right\} \text {. }
$$

Taking the union bound we conclude

$$
\mathbb{P}\left(\left(Z \cup \mathbb{Z}_{n, \varepsilon p}\right) \nrightarrow(k-\mathrm{AP})_{2}\right) \leqslant|\mathcal{C}| \exp \left\{-\eta^{2} p n / 4\right\}
$$

which goes to zero as $n$ goes to infinity. This, however, contradicts property ( $b$ ) of Corollary 5 .

\section{Proofs of the Lemmas 6 and 10}

In this section we prove the lemmas introduced in the previous section. We start with some technical observations. Given $B \subset \mathbb{Z} / n \mathbb{Z}$ and an element $z \in(\mathbb{Z} / n \mathbb{Z}) \backslash B$ let

$$
\mathcal{P}(z, B)=\{P \subset \mathbb{Z} / n \mathbb{Z} \text { : There is a } b \in B \text { such that } P \cup\{z, b\} \text { forms a } k \text {-AP }\}
$$

and let $\mathcal{P}\left(z, z^{\prime}, B\right)=\mathcal{P}(z, B) \times \mathcal{P}\left(z^{\prime}, B\right)$ where $z$ and $z^{\prime}$ need not be distinct. Further, let $\mathcal{P}(z, B, \mathbb{Z} / n \mathbb{Z})=\bigcup_{x \in \mathbb{Z} / n \mathbb{Z}} \mathcal{P}(z, B+x)$ and in the same manner define $\mathcal{P}\left(z, z^{\prime}, B, \mathbb{Z} / n \mathbb{Z}\right)$.

Fact 12. Let $z, z^{\prime} \in \mathbb{Z} / n \mathbb{Z}$ and $a \in \mathbb{Z} / n \mathbb{Z}$ be given. Then

(1) the number of $P \in \mathcal{P}(z, B, \mathbb{Z} / n \mathbb{Z})$ such that $a \in P$ is at most $k^{3}|B|$.

(2) the number of pairs $\left(P, P^{\prime}\right) \in \mathcal{P}\left(z, z^{\prime}, B, \mathbb{Z} / n \mathbb{Z}\right)$ such that $a \in P \cup P^{\prime}$ is at most $2 k^{5}|B|^{2}$.

Proof. We only prove the second property. For that we count the number of pairs $\left(P, P^{\prime}\right) \in$ $\mathcal{P}\left(z, z^{\prime}, B, \mathbb{Z} / n \mathbb{Z}\right)$ such that $a$ is in, say, $P$. Recall that there must exist $x \in \mathbb{Z} / n \mathbb{Z}$ and $b, b^{\prime} \in B+x$ such that $P \cup\{z, b\}$ and $P^{\prime} \cup\left\{z^{\prime}, b^{\prime}\right\}$ are both $k$-APs. Choosing the positions of $z$ and $a$ uniquely determines the first $k$-AP. There are at most $(k-2)$ choices for $b$ to be contained in the $k$-AP and at most $|B|$ choices of $x$ such that $b \in B+x$. Each such choice determines $P$ and moreover, gives rise to at most $|B|$ choices for $b^{\prime}$. Choosing the positions of $b^{\prime}$ and $z^{\prime}$ then determines the second $k$-AP, hence also $P^{\prime}$.

We define

$$
\mathcal{P}_{0}(z, B)=\{P \in \mathcal{P}(z, B): P \text { and } B \text { are disjoint }\}
$$

and

$$
\mathcal{P}_{0}\left(z, z^{\prime}, B\right)=\left\{\left(P, P^{\prime}\right) \in \mathcal{P}\left(z, z^{\prime}, B\right): P \cup\{z\}, P^{\prime} \cup\left\{z^{\prime}\right\} \text { and } B \text { are pairwise disjoint }\right\} .
$$

Further, let

$$
\mathcal{P}_{1}(z, B)=\mathcal{P}(z, B) \backslash \mathcal{P}_{0}(z, B) \quad \text { and } \quad \mathcal{P}_{1}\left(z, z^{\prime}, B\right)=\mathcal{P}\left(z, z^{\prime}, B\right) \backslash \mathcal{P}_{0}\left(z, z^{\prime}, B\right) .
$$

For given sets $A, B \subset \mathbb{Z} / n \mathbb{Z}$ we call $x \in \mathbb{Z} / n \mathbb{Z}$ bad (with respect to $A$ and $B$ ) if 


\section{SHARP THRESHOLD FOR VAN DER WAERDEN'S THEOREM IN RANDOM SUBSETS}

(1) there are $z \in A \backslash B+x$ and $P \in \mathcal{P}_{1}(z, B+x)$ such that $P \cup\{z\} \subset A \cup B+x$ or

(2) there are $z, z^{\prime} \in A \backslash B+x$ and $\left(P, P^{\prime}\right) \in \mathcal{P}_{1}\left(z, z^{\prime}, B+x\right)$ such that $P \cup P^{\prime} \cup\left\{z, z^{\prime}\right\} \subset A \cup B+x$.

Fact 13. Let $p \leqslant c_{1} n^{-1 /(k-1)}$ and let $B$ be a set of constant size. Let $Y_{n}$ be the set of bad elements with repect to $\mathbb{Z}_{n, p}$ and B. Then a.a.s. $\left|Y_{n}\right|<p n \log n$.

Proof. We will show that the expected size of $Y_{n}$ is of order $p n$ so that the statement follows from Markov's inequality.

For a fixed $x$ we first deal with the case that $x$ is bad due to the first property, i.e. there is a $k$-AP $P \cup\{z, b\}$ with $b \in B+x, P$ intersecting $B+x$ and $z \in \mathbb{Z}_{n, p}$ which does not belong to $B+x$. Note that after choosing $b$, one common element of $P$ and $B+x$ and their positions the $k$-AP is uniquely determined. Then there are at most $(k-2)$ choices for $z$ each of which uniquely determines one $P$. Hence the probability that $x$ is bad due to the first property is at most $|B| k^{3} p$ and summing over all $x$ we conclude that the expected number of bad elements due to the first property is at most $|B| k^{3} p n$.

If $x$ is bad due to the second property then there are two $k$-APs $P \cup\{z, b\}$ and $P^{\prime} \cup\left\{z^{\prime}, b^{\prime}\right\}$ such that two of the three sets $P \cup\{z\}, P^{\prime} \cup\left\{z^{\prime}\right\}, B+x$ intersect and $P \cup P^{\prime} \cup\left\{z, z^{\prime}\right\} \subset \mathbb{Z}_{n, p} \cup B+x$ where $z$ and $z^{\prime}$ are not in $B+x$.

We distinguish two cases and first consider all tuples $\left(P, P^{\prime}, z, z^{\prime}, b, b^{\prime}\right)$ with the above mentioned properties such that $P$ (or $P^{\prime}$ respectively) does not intersect $B+x$. Note that with this additional property the probability that $x$ is bad due to $\left(P, P^{\prime}, z, z^{\prime}, b, b^{\prime}\right)$ is at most $p^{k}$ since $z, z^{\prime}$ and $P$ all need to be in $\mathbb{Z}_{n, p}$. First, we count the number of such tuples with the additional property that $P^{\prime}$ (or $P$ respectively) also has empty intersection with $B+x$. This implies that $P$ and $P^{\prime}$ must intersect and in this case, choosing $b, b^{\prime} \in B+x$ and one common element $a \in P \cap P^{\prime}$ and the positions of $b, b^{\prime}, a$ in the $k$-AP's uniquely determines both $k$-APs. After these choices there are at most $k^{2}$ choices for $z, z^{\prime}$. Hence, there are at most $|B|^{2} k^{5} n$ such tuples for a fixed $x$.

Next, we count the number of tuples $\left(P, P^{\prime}, z, z^{\prime}, b, b^{\prime}\right)$ with the property that $P^{\prime}$ and $B+x$ intersect. In this case choosing $b^{\prime} \in B+x$ and one element in $P^{\prime} \cap B+x$ and their positions in the $k$-AP uniquely determines the second $k$-AP. Choosing $b \in B+x$, another element $a \in \mathbb{Z} / n \mathbb{Z}$, and their positions determines the first $k$-AP. After these choices there are at most $k^{2}$ choices for $z, z^{\prime}$ hence in total there are at most $|B|^{3} k^{6} n$ such tuples for a fixed $x$. We conclude that the expected number of bad $x$ due to tuples $\left(P, P^{\prime}, z, z^{\prime}, b, b^{\prime}\right)$ such that $P$ (or $P^{\prime}$ respectively) does not intersect $B+x$ is at most $p^{k} 2 n\left(|B|^{2} k^{5} n+|B|^{3} k^{6} n\right)$.

It is left to consider the tuples $\left(P, P^{\prime}, z, z^{\prime}, b, b^{\prime}\right)$ such that $\left(P, P^{\prime}\right) \in \mathcal{P}_{1}\left(z, z^{\prime}, B+x\right)$ and $P$ and $P^{\prime}$ both intersect $B+x$. In this case choosing $b, b^{\prime}$, the element(s) in $P \cap B+x$ and $P^{\prime} \cap B+x$ and their positions uniquely determine the two $k$-APs. Since $z, z^{\prime} \in \mathbb{Z}_{n, p}$ with probability $p^{2}$ the expected number of bad $x$ due to tuples $\left(P, P^{\prime}, z, z^{\prime}, b, b^{\prime}\right)$ with the above mentioned property is at most $|B|{ }^{4} k^{6} p^{2} n$.

Hence the expected size of $Y_{n}$ is

$$
\left|Y_{n}\right|<|B| k^{3} p n+2 p^{k} n\left(|B|^{2} k^{5} n+|B|^{3} k^{6} n\right)+|B|^{4} k^{6} p^{2} n<6|B|^{3} k^{6} p^{k} n^{2},
$$

as claimed. 


\section{Ehud Friedgut, Hiep Han, Yury Person, And Mathias Schacht}

Fact 14. Let $\ell \geqslant 1$ be an integer and let $F$ be an $\ell$-uniform hypergraph on the vertex set $\mathbb{Z} / n \mathbb{Z}$ which has maximum vertex degree at most $D$. Let $U=\mathbb{Z}_{n, p}$ with $p=c n^{-1 /(k-1)}$. Then with probability at most $2 D n^{-4}$ we have $e(F[U])>5 D\left(p^{\ell} n / \ell+\log n\right)$.

Proof. For $\ell=1$ the bound directly follows from Chernoff's bound

$$
\mathbb{P}(|X-\mathbb{E}(X)|>t) \leqslant 2 \exp \left\{-\frac{t^{2}}{2(\mathbb{E}(X)+t / 3)}\right\}
$$

for a binomial distributed random variable $X$. For $\ell>1$ we split the edges of $F$ into $i_{0} \leqslant D$ matchings $M_{1}, \ldots, M_{i_{0}}$, each of size at most $n / \ell$. For an edge $e \in E(F)$ let $t_{e}$ denote the random variable indicating that $e \in E(F[U])$. Then $\mathbb{P}\left(t_{e}=1\right)=p^{\ell}$ and we set $s=4 \max \left\{p^{\ell} n / \ell, \log n\right\}$. By Chernoff's bound we have

$$
\mathbb{P}\left(\exists i \leqslant i_{0}: \sum_{e \in M_{i}} t_{e}>s\right) \leqslant 2 D n^{-4} .
$$

This finishes the proof since $e(F[U])=\sum_{i \in\left[i_{0}\right]} \sum_{e \in M_{i}} t_{e}$ which exceeds $D s$ with probability at most $2 D n^{-4}$.

\section{Proof of Lemma 6 and Lemma 10}

Based on the preparation from above we give the proof of Lemma 6 and Lemma 10 in this section.

Proof of Lemma 6 . Let $t_{i}$ be the indicator random variable for the event $i \in \mathbb{Z}_{n, p}$. Consequently, $v(H)=\sum_{i \in \mathbb{Z} / n \mathbb{Z}} t_{i}$ is binomially distributed and the first property directly follows from Chernoff's bound (4).

For the second and third properties we first consider all elements from $\mathbb{Z} / n \mathbb{Z}$ which are bad with respect to $\mathbb{Z}_{n, p}$ and $B$. By Fact 13 we know that a.a.s. the set $Y_{n}$ of bad elements has size at most $n^{1-1 /(k-1)} \log n$ and in the following we will condition on this event.

We consider the degree and co-degree in the hypergraph $H\left(\mathbb{Z}_{n, p}, B,(\mathbb{Z} / n \mathbb{Z}) \backslash Y_{n}\right)$. Let $z, z^{\prime} \in \mathbb{Z} / n \mathbb{Z}$ be given. If $z$ is contained in an edge $M_{x}=M\left(\mathbb{Z}_{n, p}, B+x\right)$ then there is an element $P \in \mathcal{P}(z, B+x)$ such that $P \subset \mathbb{Z}_{n, p} \cup B+x$. It is sufficient to focus on those $P \in \mathcal{P}_{0}(z, B+x)$ since after removing $Y_{n}$ those $P \in \mathcal{P}_{1}(z, B+x)$ will have no contribution. Hence we can assume $P \subset \mathbb{Z}_{n, p}$ or equivalently $\prod_{i \in P} t_{i}=1$. Further, there are at most three different values of $y$ such that $P$ is contained in $\mathcal{P}_{0}(z, B+y)$. Hence, letting $\mathcal{P}_{0}(z, B, \mathbb{Z} / n \mathbb{Z})=\bigcup_{x \in \mathbb{Z} / n \mathbb{Z}} \mathcal{P}_{0}(z, B+x)$ we can bound the degree of $z$ by

$$
\operatorname{deg}(z) \leqslant 3 \sum_{P \in \mathcal{P}_{0}(z, B, \mathbb{Z} / n \mathbb{Z})} \prod_{i \in P} t_{i} .
$$

Similarly, if $z, z^{\prime}$ are contained in an edge $M_{x}$ then there is a pair $\left(P, P^{\prime}\right) \in \mathcal{P}_{0}\left(z, z^{\prime}, B+x\right)$ such that $P \cup P^{\prime} \subset \mathbb{Z}_{n, p} \cup B+x$, i.e. $\prod_{i \in P \cup P^{\prime}} t_{i}=1$. We obtain

$$
\operatorname{codeg}\left(z_{1}, z_{2}\right) \leqslant 9 \sum_{\left(P, P^{\prime}\right) \in \mathcal{P}_{0}\left(z, z^{\prime}, B, \mathbb{Z} / n \mathbb{Z}\right)} \prod_{i \in P \cup P^{\prime}} t_{i}
$$




\section{SHARP THRESHOLD FOR VAN DER WAERDEN'S THEOREM IN RANDOM SUbSETS}

We consider $\mathcal{P}_{0}(z, B, \mathbb{Z} / n \mathbb{Z})$ (respectively, $\mathcal{P}_{0}\left(z, z^{\prime}, B, \mathbb{Z} / n \mathbb{Z}\right)$ ) as a $(k-2)$-uniform (resp., $(2 k-4)$-uniform) hypergraph on the vertex set $\mathbb{Z} / n \mathbb{Z}$. By Fact 12 we know that the maximum degree of the hypergraph is at most $k^{3} K$ (resp. $5 k^{5} K^{2}$ ). By Fact 14 the probability that $\operatorname{deg}(z)>10 k^{3} K p^{k-2} n$ or $\operatorname{codeg}\left(z, z^{\prime}\right)>8 \log n$ is at most $2 k^{3} K n^{-4}$. Taking the union bound over all elements and all pairs of $\mathbb{Z} / n \mathbb{Z}$ we obtain the desired property.

Proof of Lemma 10. For given $k, c, K$ and $\alpha$ set $L=20 c^{k-1} k^{2} / \alpha$ and $\alpha^{\prime}=\alpha / 2(L K)^{L}$ and let some linear order on $\mathbb{Z} / n \mathbb{Z}$ be given. First we choose $Y_{n}$ so as to guarantee regularity of the triple $\left(\mathbb{Z}_{n, p}, B,(\mathbb{Z} / n \mathbb{Z}) \backslash Y_{n}\right)$. Note that there are two sources of irregularity: $k$-APs containing two elements from $B+x$ for some $x \in \mathbb{Z} / n \mathbb{Z}$ and pairs of $k$-APs with one common element in $\mathbb{Z}_{n, p}$ and each containing one element in $B+x$ for some $x \in \mathbb{Z} / n \mathbb{Z}$. These are ruled out by removing all $x$ which are bad with respect to $\mathbb{Z}_{n, p}$ and $B$. By Fact 13 a.a.s. the set $Y_{n}$ of bad elements has size at most $n^{1-1 /(k-1)} \log n$.

Further, we count the number of $(k-1)$-element sets in $\mathbb{Z}_{n, p}$ which arise from $k$-APs with one element removed. The expected number of such sets is at most $p^{k-1} k n^{2}$ and the variance is of order at most $p^{k-2} n^{2}$. Hence, by Chebyshev's inequality the number of such sets in $\mathbb{Z}_{n, p}$ is at most $2 c^{k-1} k n$ asymptotically almost surely.

Consider any set $A \subset \mathbb{Z} / n \mathbb{Z}$ which posseses the two properties mentioned above: there is a set $Y_{n}$ of size at most $n^{1-1 /(k-1)} \log n$ such that $\left(A, B,(\mathbb{Z} / n \mathbb{Z}) \backslash Y_{n}\right)$ is regular and the number of $(k-1)$-element sets in $A$ which arise from $k$-APs with one element removed is at most $2 c^{k-1} k n$. Let $X \subset \mathbb{Z} / n \mathbb{Z}$ of size $|X| \geqslant \alpha n$ be given. For every $x \in X \backslash Y_{n}$ let $\ell_{x}$ denote the size of $M_{x}=M(A, B, X)$. Then there are at least $\ell_{x} /(k-1)$ sets of size $(k-1)$ contained in $M_{x}$ each forming a $k$-AP with an element in $B+x$. Further, each such set is contained in $B+x^{\prime}$ for at most three $x^{\prime} \in \mathbb{Z} / n \mathbb{Z}$, hence, $\sum_{x \in X \backslash Y_{n}} \ell_{x} /(k-1) \leqslant 6 c^{k-1} k n$ and we conclude that the number of $x \in X \backslash Y_{n}$ such that $\ell_{x}>20 c^{k-1} k^{2} / \alpha=L$ is at most $\alpha n / 3$. Moreover, there are at most $|B|^{\ell}$ distinct profiles of length $\ell$, hence there is a profile $\pi$ of length $\ell \leqslant L$ and a set $U \subset X \backslash Y_{n}$ of size $|U| \geqslant \alpha n / 2 K^{L}$ such that $(A, B, U)$ has profile $\pi$.

To obtain a set $X^{\prime} \subset U$ such that $\left(A, B, X^{\prime}\right)$ is index consistent we consider a random partition of $A$ into $\ell$ classes $\left(V_{1}, \ldots, V_{\ell}\right)$. We say that an edge $M_{x} \in H(A, B, U)$ with the elements $z_{1}<\cdots<z_{\ell}$ survives if $z_{i} \in V_{i}$ for all $i \in[\ell]$. The probability of survival is $\ell^{\ell}$, hence there is a partition such that at least $|U| / \ell^{\ell}$ edges survives. Choosing the corresponding set $X^{\prime} \subset U$ yields a set with the desired properties.

\section{Proof of Lemma 8}

In this section we prove Lemma 8. The proof relies crucially on a structural theorem of Balogh, Morris and Samotij [1] which we state in the following. Let $H$ be a uniform hypergraph with vertex set $V$ and let $\mathcal{F}$ be an increasing family of subsets of $V$ and $\varepsilon \in(0,1]$. The hypergraph $H$ is called $(\mathcal{F}, \varepsilon)$-dense if for every $A \in \mathcal{F}$

$$
e(H[A]) \geqslant \varepsilon e(H) .
$$

Further, let $\mathcal{J}(H)$ denote the set of all independent sets of $H$. The so-called container theorem by Balogh, Morris and Samotij [1, Theorem 2.2] reads as follows. 


\section{Ehud Friedgut, Hiep Han, Yury Person, And Mathias Schacht}

Theorem 15 (Container theorem). For every $\ell \in \mathbb{N}$ and all positive $c$ and $\varepsilon$, there exists a positive constant $c^{\prime}$ such that the following holds. Let $H$ be an $\ell$-uniform hypergraph and let $\mathcal{F}$ be an increasing family of subsets of $V$ such that $|A| \geqslant \varepsilon v(H)$ for all $A \in \mathcal{F}$. Suppose that $H$ is $(\mathcal{F}, \varepsilon)$-dense and $p \in(0,1)$ is such that for every $t \in[\ell]$ the maximum $t$-degree $\Delta_{t}(H)$ of $H$ satisfies

$$
\Delta_{t}(H)=\max _{\substack{T \subseteq V \\|T|=t}}\{|\{e \supseteq T: e \in E(H)\}|\} \leqslant c p^{t-1} \frac{e(H)}{v(H)} .
$$

Then there is a family $\mathcal{S} \subseteq\left(\begin{array}{c}V(H) \\ \leqslant c^{\prime} p v(H)\end{array}\right)$ and functions $f: \mathcal{S} \rightarrow \overline{\mathcal{F}}$ and $g: \mathcal{J}(H) \rightarrow \mathcal{S}$ such that for every $I \in \mathcal{J}(H)$,

$$
g(I) \subseteq I \quad \text { and } \quad I \backslash g(I) \subseteq f(g(I)) .
$$

Theorem 15 roughly says that if an uniform hypergraph $H$ satisfies certain conditions then the set of independent sets $\mathcal{J}(H)$ of $H$ can be "captured" by a family $\mathcal{S}$ consisting of small sets. Indeed, every independent set $I \in \mathcal{J}(H)$ contains a (small) set $g(I) \in \mathcal{S}$ and the remaining elements of $I$ must come from a set determined by $g(I)$.

We are now in a position to derive Lemma 8 from Theorem 15.

Proof of Lemma 8. Given the constants $k, \ell, C_{0}$ and $C_{1}$ we apply Theorem 15 with $\varepsilon=1 / 2$, and $c=\max \left\{1, C_{1} / C_{0}\right\}$ to obtain $c^{\prime}$. We let $C^{\prime}=c^{\prime}$ and $\beta=\min \left\{1 / 4, C_{0} /\left(4 C_{1}\right)\right\}>0$.

We define the increasing family $\mathcal{F}$ by

$$
\mathcal{F}=\{A: A \subset V(H) \text {, s.t. }|A| \geqslant m / 2 \text { and } e(H[A]) \geqslant e(H) / 2\} .
$$

Clearly, $H$ is $(\mathcal{F}, 1 / 2)$-dense and, moreover, any set $A \in \overline{\mathcal{F}}$ has size less than $m / 2$ or we have $e(H[A])<e(H) / 2$. Thus, at least $e(H) / 2$ edges of $H$ are incident to the vertices outside of $A$. Therefore, $\Delta_{1}(H)(m-|A|) \geqslant e(H) / 2 \geqslant C_{0} m^{1+1 /(k-2)} / 2$ and with $\Delta_{1}(H) \leqslant C_{1} m^{1 /(k-2)}$ we conclude:

$$
|A| \leqslant(1-2 \beta) m
$$

We define $p=m^{-1 /(k-2)(\ell-1)}$ so that $\Delta_{t}(H) \leqslant c p^{t-1} \frac{e(H)}{v(H)}$ for all $t \in[\ell]$. In fact, for $t=1$ this follows directly from the bound $\Delta_{1}(H) \leqslant C_{1} m^{\frac{1}{k-2}}$ given by the assumption of Lemma 8 . For $t=2, \ldots, \ell-1$ we use $\Delta_{t}(H) \leqslant \Delta_{2}(H)$ and on $\Delta_{2}(H)$ given by the assumption of Lemma 8 . Finally, for $t=\ell$ we note that $\Delta_{\ell}(H)=1$ and the desired bound follows again from the choices of $p$ and $c$.

Thus there exist a family $\mathcal{S}$ and functions $f: \mathcal{S} \rightarrow \overline{\mathcal{F}}$ and $g: \mathcal{J}(H) \rightarrow \mathcal{S}$ with the properties described in Theorem 15. We define

$$
\mathcal{A}=\{S \cup f(S): S \in \operatorname{Im}(g)\}
$$

where $\operatorname{Im}(g)$ is the image of $g$. Our cores will be the complements of the elements of $\mathcal{A}$,

$$
\mathcal{C}=\{V(H) \backslash A: A \in \mathcal{A}\} .
$$


Since $|\mathcal{C}|=|\mathcal{A}| \leqslant|\mathcal{S}|$, we infer $(i)$ of Lemma 8. Further, every $A \in \mathcal{A}$ has size at most

$$
(1-2 \beta) m+C^{\prime} p m \leqslant(1-\beta) m
$$

which yields the property $(i i)$ of Lemma 8 .

Finally, by the properties of the functions $f$ and $g$, every independent set $I$ is contained in $A=g(I) \cup f(g(I))$, so, by taking complements, every hitting set contains an element of $\mathcal{C}$ which completes the property (iii) of Lemma 8.

\section{Proof of Lemma 11}

In this section we prove Lemma 11 which relies on the following result by the last author [12] (see also $[1,2,11])$.

Theorem 16. For every integer $k \geqslant 3$ and every $\gamma \in(0,1)$ there exists $C$ and $\xi>0$ such that for every sequence $p=p_{n} \geqslant C n^{-1 /(k-1)}$ the following holds a.a.s. Every subset of $\mathbb{Z}_{n, p}$ of size at least $\gamma p n$ contains at least $\xi p^{k} n^{2}$ arithmetic progression of length $k$.

With this result at hand we now prove Lemma 11.

Proof of Lemma 11. The upper bound on the size of $\mathbb{Z}_{n, p}$ follows from Chernoff's bound (4). For the second property let $k \geqslant 3$ and $\gamma$ be given. For $k \geqslant 4$ we apply Theorem 16 with $k-1$ and $\gamma$ to obtain $C$ and $\xi$. We may assume that $\xi \leqslant \gamma^{2} / 4$ and we note that in the case $k \geqslant 4$ we have $p \geqslant c_{0} n^{-1 /(k-1)}>C n^{-1 /(k-2)}$ for sufficiently large n. We choose $\delta=\xi^{2} / 20$.

Let $S \subset \mathbb{Z}_{n, p}$ be a set of size at least $\gamma p n$. For a given $i \in \mathbb{Z} / n \mathbb{Z}$ let $\operatorname{deg}(i)$ denote the number of $(k-1)$-APs in $S$ which form a $k$-AP with $i$. Note that $i \in F(S)$ if $\operatorname{deg}(i) \neq 0$. Then a.a.s we have

$$
\sum_{i \in \mathbb{Z} / n \mathbb{Z}} \operatorname{deg}(i) \geqslant \xi p^{k-1} n^{2}
$$

which holds trivially for the case $k=3$ due to $\xi \leqslant \gamma^{2} / 4$ and which is a consequence of Theorem 16 for larger $k$.

Further, let $W=\sum_{i \in \mathbb{Z} / n \mathbb{Z}}\left(\begin{array}{c}\operatorname{deg}(i) \\ 2\end{array}\right)$. Then, as $S \subset \mathbb{Z}_{n, p}$

$$
\mathbb{E}(W) \leqslant n\left(p^{2(k-1)} n^{2}+k^{2} p^{k-1-\left\lfloor\frac{k-1}{2}\right\rfloor} n\right) \leqslant 2 p^{2(k-1)} n^{3}
$$

and its variance is of order at most $p^{2(k-1)} n^{3}$. Hence, by Chebyshev's inequality we have $\sum_{i \in \mathbb{Z} / n \mathbb{Z}}\left(\begin{array}{c}\operatorname{deg}(i) \\ 2\end{array}\right) \leqslant 4 p^{2(k-1)} n^{3}$ asymptotically almost surely.

Altogether we obtain

$$
\left(\begin{array}{c}
\xi p^{k-1} n^{2} \\
2
\end{array}\right) \leqslant\left(\begin{array}{c}
\sum_{i \in F(S)} \operatorname{deg}(i) \\
2
\end{array}\right) \leqslant|F(S)| \sum_{i \in F(S)}\left(\begin{array}{c}
\operatorname{deg}(i) \\
2
\end{array}\right) \leqslant|F(S)| 4 p^{2(k-1)} n^{3}
$$

and we conclude that $|F(S)| \geqslant \xi^{2} n / 20=\delta n$. 


\section{Ehud Friedgut, Hiep Han, Yury Person, and Mathias Schacht}

\section{Acknowledgement}

We thank the anonymous referee for her or his constructive and helpful remarks.

\section{References}

[1] J. Balogh, R. Morris, and W. Samotij, Independent sets in hypergraphs, J. Amer. Math. Soc. 28 (2015), no. 3, 669-709, DOI 10.1090/S0894-0347-2014-00816-X. MR3327533 个1, 3, 7, 9, 15, 17

[2] D. Conlon and W. T. Gowers, Combinatorial theorems in sparse random sets, available at arXiv:1011.4310. Submitted. $\uparrow 1,17$

[3] D. Conlon, W. T. Gowers, W. Samotij, and M. Schacht, On the KŁR conjecture in random graphs, Israel J. Math. 203 (2014), no. 1, 535-580, DOI 10.1007/s11856-014-1120-1. MR3273450 ^1

[4] E. Friedgut, Sharp thresholds of graph properties, and the k-sat problem, J. Amer. Math. Soc. 12 (1999), no. 4, 1017-1054, DOI 10.1090/S0894-0347-99-00305-7. With an appendix by Jean Bourgain. MR1678031 (2000a:05183) $\uparrow 3$

[5] _ Hunting for sharp thresholds, Random Structures Algorithms 26 (2005), no. 1-2, 37-51, DOI 10.1002/rsa.20042. MR2116574 (2005k:05216) $\uparrow 3$

[6] E. Friedgut and M. Krivelevich, Sharp thresholds for certain Ramsey properties of random graphs, Random Structures Algorithms 17 (2000), no. 1, 1-19, DOI 10.1002/1098-2418(200008)17:1<1::AID-RSA1>3.0.CO;2-4. MR1768845 (2001i:05136) $\uparrow 2$

[7] E. Friedgut, V. Rödl, A. Ruciński, and P. Tetali, A sharp threshold for random graphs with a monochromatic triangle in every edge coloring, Mem. Amer. Math. Soc. 179 (2006), no. 845, vi+66, DOI 10.1090/memo/0845. MR2183532 (2006h:05208) $\uparrow 2,3$

[8] E. Friedgut, V. Rödl, and M. Schacht, Ramsey properties of random discrete structures, Random Structures Algorithms 37 (2010), no. 4, 407-436, DOI 10.1002/rsa.20352. MR2760356 (2012a:05274) 11

[9] V. Rödl and A. Ruciński, Threshold functions for Ramsey properties, J. Amer. Math. Soc. 8 (1995), no. 4, 917-942, DOI 10.2307/2152833. MR1276825 (96h:05141) $\uparrow 1,2$

[10] , Rado partition theorem for random subsets of integers, Proc. London Math. Soc. (3) 74 (1997), no. 3, 481-502, DOI 10.1112/S0024611597000178. MR1434440 (98d:05147) $\uparrow 2,3,4,6$

[11] D. Saxton and A. Thomason, Hypergraph containers, Invent. Math. 201 (2015), no. 3, 925-992, DOI 10.1007/s00222-014-0562-8. MR3385638 ^1, 3, 17

[12] M. Schacht, Extremal results for random discrete structures. Submitted. $\uparrow 1,17$

[13] M. Schacht and F. Schulenburg, Sharp thresholds for Ramsey properties of strictly balanced nearly bipartite graphs, available at arXiv:1602.02501. Submitted. $\uparrow 3$

[14] P. Varnavides, On certain sets of positive density, J. London Math. Soc. 34 (1959), 358-360. MR0106865 (21 \#5595) $\uparrow 11$

[15] B. L. van der Waerden, Beweis einer Baudetschen Vermutung, Nieuw Arch. Wiskd., II. Ser. 15 (1927), 212-216 (German). $\uparrow 2$ 


\section{AUTHORS}

Ehud Friedgut

Faculty of Mathematics and Computer Science

Weizmann Institute of Science

Rehovot, Israel

ehud.friedgut@weizmann.ac.il

\section{Hiệp Hàn}

Instituto de Matemáticas

Pontificia Universidad Católica de Valparaíso

Valparaíso, Chile

han.hiep@googlemail.com

Yury Person

Institut für Mathematik

Goethe-Universität

Frankfurt am Main, Germany

person@math.uni-frankfurt.de

http://www.math.uni-frankfurt.de/ person/

Mathias Schacht

Fachbereich Mathematik

Universität Hamburg

Hamburg, Germany

schacht@math.uni-hamburg.de

http://www .math.uni-hamburg.de/home/schacht/ 